\title{
Treatment improvement of pulmonary epithelioid hemangioendothelioma: A case report.
}

\author{
Yalei Lv, Xue Zhang, Wei Liu*
}

Department of Medical Oncology, the Fourth Hospital of Hebei Medical University, Shijiazhuang, Hebei, PR China

\begin{abstract}
Pulmonary Epithelioid Hemangioendothelioma (PEH) is one rather rare low-grade malignant tumor. Owing to its relatively low incidence, standard treatments on PEH are relatively less. A 52 y old male PEH patient treated with endostatin had received outstanding clinical outcome, considering his brain metastases and pleural effusion were both negative effects upon prognosis. Patient's pathological characteristics, iconographic characteristics would play an essential role on his response to clinical treatment and prognosis. So far, standard treatments upon PEH are still not available, and the inhibition of tumor growth can be achieved through inhibition of tumor angiogenesis and interrupting cellular nutritious supply.
\end{abstract}

Keywords: Tumor, PEH, Endostar, Treatment, Prognosis.

Accepted on July 19, 2017

\section{Introduction}

Malignant vascular tumors can be often quite challenging, it is quite difficult to distinguish an Epithelioid Hemangioendothelioma (EHE) from an Epithelioid Hemangioma $(\mathrm{EH})$ or between an Epithelioid Angiosarcoma (EA) and a malignant EHE [1]. Representing less than $1 \%$ of all vascular tumors, EHE is a rare vascular tumor with an epithelioid and histiocytoid appearance that originates from vascular endothelial or pre-endothelial cells [2].

Pulmonary Epithelioid Hemangioendothelioma (PEH) is an uncommon lung malignancy of endothelial origin [3] which was first described in 1983 [4] and it normally happen as a bilateral multiple nodules in young females $[5,6]$.

Some cases initially presenting small nodular densities at the bases of the lungs followed by bilateral pleural effusions [7]. Some case with the initial manifestation as a pseudoaneurysm caused by the rupture of the right pulmonary artery after tumor invasion and the diagnosis was confirmed by the morphologic and immunocytochemical features after surgery [8].

Besides demonstrating unpredictable presentation features and prognosis, the paucity of established treatment guidelines remains a challenge in managing these patients [9].

Wide surgery is probably associated with a better prognosis [10]. Some were treated with pazopanib as first line [11]. A recently reported protocol of 33 radiation fractions totalling 5,940 cGy was also applied and in $8 \mathrm{y}$ after surgery and RT revealed neither recurrence nor any change [12].

\section{Case Report}

A 52 y heavy-smoking male Chinese was registered with pulmonary nodules in December 2012. The patient has hypertension, hyperlipemia and hypercholesterolemia, and was treated with zocor and metoprolol for $15 \mathrm{y}$. The period before registration, he had experienced weight loss, dry cough and chest pain. And highly metabolize bilateral pulmonary nodules were found from PET-CT (Figure 1) and CT (Figure 2A) with no other specific symptoms. With consideration of his physical conditions, surgical resection was performed on December $17^{\text {th }}$ 2012, and three circular, medium hard goiters were removed from the tip section of his left lung. According to the IHC results of tumor tissue, the patient harbored positive CD 34 and CD 31. It is hard to make an accurate diagnosis according to the pathological characteristics. This study was conducted in accordance with the declaration of Helsinki. This study was conducted with approval from the Ethics Committee of the Fourth Hospital of Hebei Medical University. Written informed consent was obtained from participant.

Considering the pathological situations and willingness of the patient, no treatment was applied upon him. ACT (Figure 2B) was applied two months after the surgery, and the results demonstrated multiple nodules of both lungs and implied metastases of tumor, enlargement of subcarinal lymph nodes, thickening of left pleural, and effusion of left pleural. Three months after the surgery, the patient took some exercise, like table tennis, and got headache, fever and unconsciousness. As a result, multiple irregular heterogeneous density of bilateral cerebral hemisphere, high density of left frontal lobe, narrowing of left ventricle, shallow or disappeared part of brain sulcus was found through $\mathrm{CT}$, and his PS has dropped from 0 
to 2 immediately. Furthermore, rupture and bleeding of metastatic brain tumor through MRI (Figure 3). After 3dimentional conformal and whole brain radiation therapy, supportive therapy has been applied and improved his symptoms, like lowering the intracranial pressure. With the appearance of soft sarcomas under his epithelial bilateral arms and left rib, other symptoms have shown up, like poor mobility, pressure pain and poor quality of his somnia. A close detection of CT was performed on April 9 2013 (Figure 2C) and found that the number and size of multiple nodules have dramatically increased and enlarged, new nodules have shown up in adrenal gland and left ribs have been destructed. Therefore, another tumor tissue was used for IHC detection, considering the results of IHC and clinical suggestions of several experts, the patient was eventually diagnosed as $\mathrm{PEH}$. Recombinant human endostatin works as anti-angiogenesis medication and succeeds in clinical treatment of non-small cell lung cancer. Since no standard treatment is available, the patient was treated with recombinant human endostatin 7.5 $\mathrm{mg} / \mathrm{m}^{2}$, d 1 14. With any luck, patient experiences relatively stable disease stage for two weeks, and the side-effect is not intolerable. In July 2013, the number of subcutaneous nodules has increased and multiple metastases were detected inside his brain. The patient died in the middle of July. The overall PFS is approximately 2 months, and his overall survival is approximately 8 months.

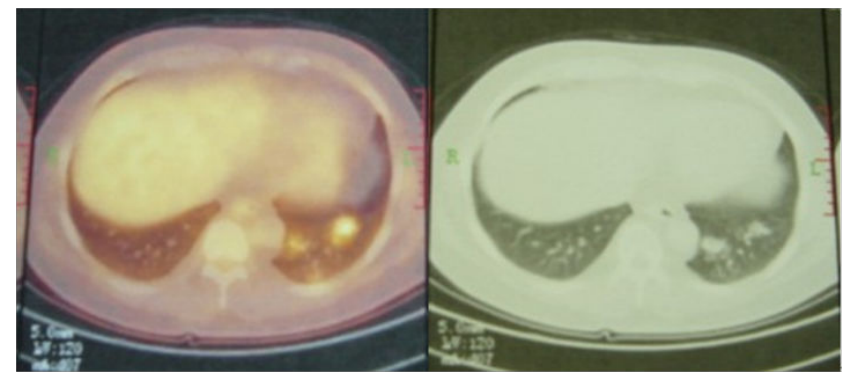

Figure 1. Metabolism status of nodes in both lungs by PET-CT.
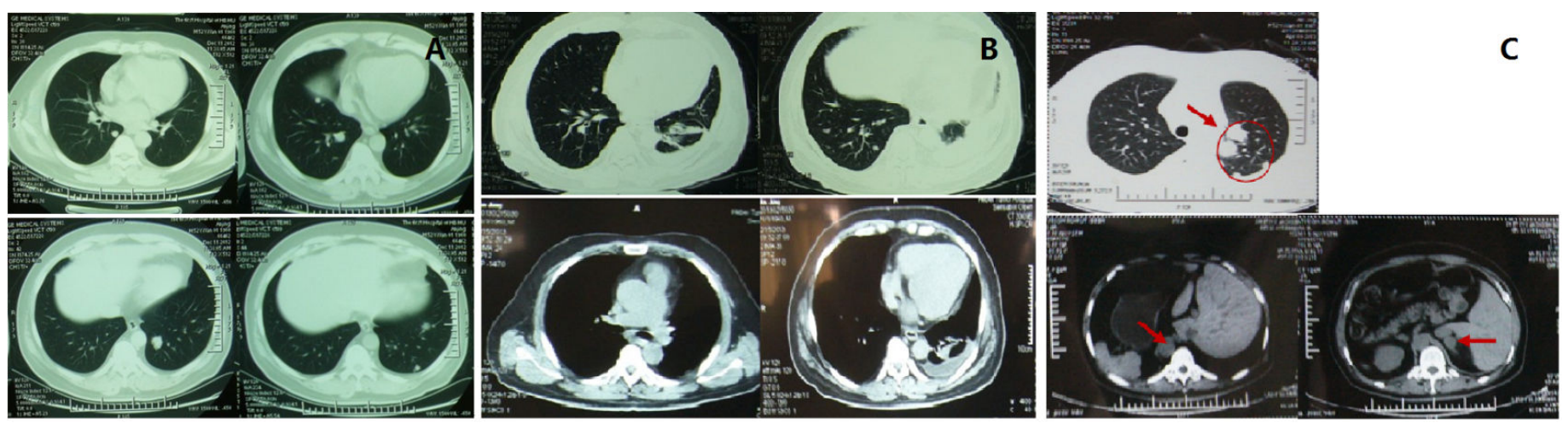

Figure 2. Multiple nodules of both lungs (A: preoperation of CT; B: 2 months postoperation of ACT; C: Most recently, 16 months after the operation, of CT).

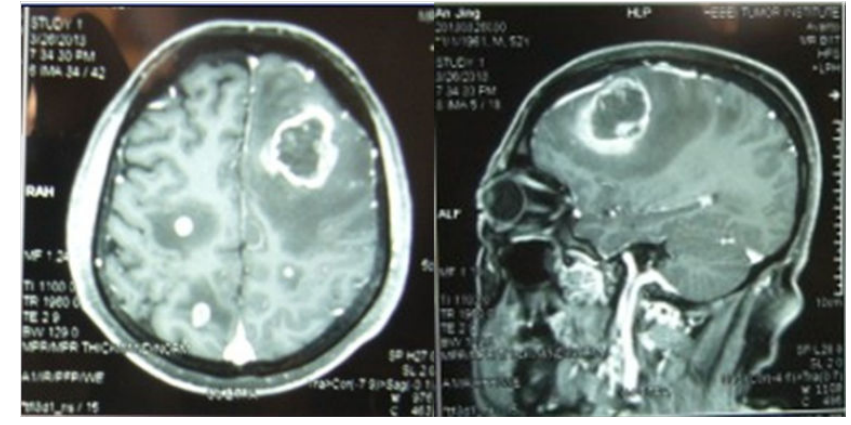

Figure 3. Brain MRI. Multiple irregular heterogeneous densities were found in both cerebral hemispheres, especially in left frontal lobe. His left ventricle narrowed, and a part of the brain sulcus fission shallow disappeared.

\section{Discussion}

Approximately half of PEHs (49.5\%) has no identifiable clinical symptoms and share some symptoms with lung cancer, such as thoracic pain (16\%), cough and mild dyspnea (18.3\%), occasional hemoptysis and weight loss (6.5\%) [6]. Almost three quarters of PEHs are female and $65 \%$ share with bilateral multiple nodular opacities [13-16], which may be a vital factor of poor prognosis. In this case, the patient was a 52 y old male with chest pain, cough and weight loss.

From the picture of his CT, small nodules diffused in both lungs and proliferated along small vessels or tracheole with clear or blurred edge $(<15 \mathrm{~mm})$ located next to the subpleural region and showed traction syndrome towards pleura. The cells of $\mathrm{PEH}$ were either round or polygon, and proliferated around vessels, resulting in forms of isolated nodules and multiple nodules, and normally non-calcification. As a matter of fact, those tumor cells share the same characteristics with epithelioid hemangioendothelioma [13-15], such as round or polygon shape, rich in transparent eosinophilic cytoplasm, cytoplasmic vacuoles and vesicular nucleus, which could play a vital role in the diagnosis of PEH. Besides, the IHC results of his tumor tissue showed positive of CD 34 and CD 31, which imply that CD 34 could play a vital role in the diagnosis of PEH [6]. Moreover, IHC results of this patient share some same characteristics with squamous cell carcinoma, like negative TTF-1 and p63 [17], which could also play a vital role in diagnosis. Therefore, both the histological characteristics of tumor cells and specific IHC results play an essential part in diagnosis of PEH. 
Normally, enlargement of mediastinal lymph nodes and occurrence of pleural effusion $[5,6,16]$ equal to poor prognosis, while age, gender and smoking history cannot be used as significant prognosis factors $[6,16,18,19]$. A review of $75 \mathrm{PEH}$ patients by Patrick et al. [5] states the median survival period of patients with blood pleural effusion would be less than one year, and this conclusion has also been proved in Asia [16]. Besides, central nervous metastasis and dyspnea seem to make a great contribution to poor prognosis $[6,18]$, and in this case, the patient has experienced enlargement of subcarinal lymph nodes and left pleural effusion, and his brain metastasis and dyspnea may be another factor that lead to rapid disease progress and poor prognosis. As a result, and three months after the resection surgery, brain metastases were found and physical status has dropped to 2, and the application of endostar could only provide patient with 2 -month PFS.

In the past six months, several case reports on $\mathrm{PEH}$ have been published, and only a few demonstrate to be self-curable [16]. The current prior therapy for single nodule from lung is always surgical resection, like wedging resection or pulmonary lobectomy. After the wedge surgery of this patient, he remained PS0 for three months, which imply surgery might be a basic primary treatment of $\mathrm{PEH}$.

Unfortunately, PEH show resistances to several chemotherapies [5,14-16,20-22]. A case report of $\mathrm{PEH}$ published in 1999 illustrates that 6 cycles of NSC-141540 and carboplatin provide this patient a good chance of Complete Response (CR) and his PFS period lasts for 18 months [20]. Another case report from Poland researchers on advanced PEH states that 3 months treatment with interferon- $\alpha$ ( 3 million units per day, $3 \mathrm{~d}$ a week) has down-regulated the size of PEH, but resulted in intolerable side-effects [5]. Some scholars believe part of lung $\mathrm{PEH}$ patients have positive hormone receptors [14], therefore, endocrine therapy could be one of the most effective therapies for those patients, especially in females, considering to the contributions of normal receptors for oestrogen and progesterone $[5,16]$. Besides, sorafenib could be a first-line treatment for PEH [19].

Moreover, since tumor cells proliferate along with the developments of vessels, applications of anti-angiogenesis drugs should theoretically provide effective treatment for $\mathrm{PEH}$. Thalidomide, as an inhibition of angiogenesis, has been reported that thalidomide has played a vital role in treatment of metastatic hepatic epithelioid hemangioendothelioma [21,22]. Considering the physical status and previous treatments of the patient, targeted therapy seems to be a better option, especially vessel targeted. Therefore, endostar was used as the only therapy, owing to the fact that bevacizumab could not have a significant influence on PEH treatment [23]. As a matter of fact, during the whole period of endostar treatment, subcutaneous nodules became less swollen and his status was stabilized for two months. With the development of tumor, the organs of his whole body went on failure and led to death. According to another case of a 40-year old male Chinese [6], endostar could only stabilize disease and not be able to contribute to minimize the size or number of nodules. The possible reasons that endostar did not help this patient long enough are the amount of endostar usage and the delayed timing of endostar treatment.

Malignant heaemangioendothelioma are normally treated with radical excision [14]. The patient was treated with radiotherapy, which probably play a vital role in the prolonged OS of this patient. Although both Chinese patients had brain metastases after surgery, the 40 y old patient treated with endostar, cisplatin, paclitaxel for 3 cycles only had a OS of 6 months while there was no sign of his pleural effusion [6], whereas, this 52 y old patient with pleural effusion treated only with half amount of endostar had a OS of approximately 8 months, which suggests radiotherapy could probably play a vital role in the treatment of PEH. Similar to this case, a Spanish PEH patient had a PD after radiotherapy and died in 18 months after the first diagnosis [18].

So far, standard treatment to PEH is not available. Although targeted therapy or combined with chemotherapy have been applied upon PEH for years, no big progress has been made. Surgical resection seems to play a fundamental part in the treatment of $\mathrm{PEH}$, while chemotherapy seems to contribute less than endocrine therapy, and vascular targeted therapy, such as endostar, seems to have an influence on disease stabilization. Besides, radiotherapy seems to play a vital role in the treatment of PEH. In a word, combination of radiation therapy with endostar after surgical resection could be an effective treatment for PEH.

\section{Acknowledgement}

Give all the thanks to staffs of our institute that have been working on the case with us. Also, give thanks to the relatives of the patient for giving permission on the case release.

\section{References}

1. Antonescu C. Malignant vascular tumors--an update. Mod Pathol 2014; 27: 30-38.

2. Sardaro A, Bardoscia L, Petruzzelli MF, Nikolaou A, Detti B, Angelelli G. Pulmonary epithelioid hemangioendothelioma presenting with vertebral metastases: a case report. J Med Case Rep 2014; 8: 201.

3. Cao Y, Zou SM, Zhang KT, Lu N, Liu Y, Feng L, Wen P, Han NJ, Lin DM. Genetic alterations in pulmonary epithelioid hemangioendothelioma and epithelioid angiosarcoma. Histol Histopathol 2011; 26: 491-496.

4. Dail DH, Liebow AA, Gmelich JT, Friedman PJ, Miyai K, Myer W, Patterson SD, Hammar SP. Intravascular, bronchiolar, and alveolar tumor of the lung (IVBAT): an analysis of twenty cases of a peculiar sclerosing endothelial tumor. Cancer 1983; 51: 452-464.

5. Bagan P, Hassan M, Le Pimpec Barthes F, Peyrard S, Souilamas R, Danel C, Riquet M. Prognostic factors and surgical indications of pulmonary epithelioid hemangioendothelioma: a review of the literature. Ann Thorac Surg 2006; 82: 2010-2013. 
6. Kumar P, Judson I, Nicholson AG, Ladas G. Mediastinal hemangioma: successful treatment by alpha-2a interferon and postchemotherapy resection. J Thorac Cardiovasc Surg 2002; 124: 404-406.

7. Sayah M, VandenBussche C, Maleki Z. Epithelioid hemangioendothelioma in pleural effusion. Diagn Cytopathol 2015; 43: 751-755.

8. Wu XN, Chen MJ, Li DQ, Hu JG, Yu FL. Pulmonary artery pseudoaneurysm caused by a rare vascular tumor: epithelioid hemangioendothelioma. Thorac Cardiovasc Surg 2014; 62: 92-94.

9. Soo CI, Ng BH, Tan EL, Abdul Hamid F. Ambiguous presentations of pulmonary epithelioid hemangioendothelioma: Two case reports of a rare pulmonary malignancy. SAGE Open Med Case Rep 2016; 4: $2050313 \times 16650323$.

10. Luzzati A, Gagliano F, Perrucchini G, Scotto G, Zoccali C. Epithelioid hemangioendothelioma of the spine: results at seven years of average follow-up in a series of 10 cases surgically treated and a review of literature. Eur Spine J 2015; 24: 2156-2164.

11. Semenisty V, Naroditsky I, Keidar Z, Bar-Sela G. Pazopanib for metastatic pulmonary epithelioid hemangioendothelioma-a suitable treatment option: case report and review of anti-angiogenic treatment options. BMC Cancer 2015; 15: 402.

12. Drazin D, Gandhi R, Slodkowska E, Boulos AS. Epithelioid hemangioendothelioma of the mastoid: resection for recurrence and adjuvant radiation with 8-year follow-up. Case Rep Surg 2013; 2013: 469201.

13. Gordillo GM, Onat D, Stockinger M, Roy S, Atalay M, Beck FM, Sen CK. A key angiogenic role of monocyte chemoattractant protein-1 in hemangioendothelioma proliferation. Am J Physiol Cell Physiol 2004; 287: 866-873.

14. Kumazawa Y, Maeda K, Ito M, Yamakawa M, Hino T, Nakamura C, Uchimura F, Arai S. Expression of glucocorticoid receptor and 11beta hydroxysteroid dehydrogenase in a case of pulmonary epithelioid haemangioendothelioma. Mol Pathol 2002; 55: 61-64.

15. Errani C, Zhang L, Sung YS, Hajdu M, Singer S, Maki RG, Healey JH, Antonescu CR. A novel WWTR1-CAMTA1 gene fusion is a consistent abnormality in epithelioid hemangioendothelioma of different anatomic sites. Genes Chromosomes Cancer 2011; 50: 644-653.
16. Kitaichi M, Nagai S, Nishimura K, Itoh H, Asamoto H, Izumi T, Dail DH. Pulmonary epithelioid haemangioendothelioma in 21 patients, including three with partial spontaneous regression. Eur Respir J 1998; 12: 89-96.

17. Conde E, Angulo B, Redondo P, Toldos O, García-García E, Suarez-Gauthier A, Rubio-Viqueira B, Marron C, Garcia-Lujan R, Sanchez-Cespedes M, Lopez-Encuentra A, Paz-Ares L, Lopez-Ríos F. The use of P63 immunohistochemistry for the identification of squamous cell carcinoma of the lung. PLoS One 2010; 5: 12209.

18. Díaz R, Segura A, Calderero V, Cervera I, Aparicio J, Jorda MV, Pellín L. Central nervous system metastases of a pulmonary epitheloid haemangioendothelioma. Eur Respir J 2004; 23: 483-486.

19. Mcculloch M, Russin M, Nachat A. Recurrence of epithelioid hemangioendothelioma during pregnancy: case report and systematic review. Perm J 2016; 20: 84-89.

20. Pinet C, Magnan A, Garbe L, Payan MJ, Vervloet D. Aggressive form of pleural epithelioid haemangioendothelioma: complete response after chemotherapy. Eur Respir J 1999; 4: 237-238.

21. Raphael C, Hudson E, Williams L, Lester JF, Savage PM. Successful treatment of metastatic hepatic epithelioid hemangioendothelioma with thalidomide: a case report. J Med Case Rep 2010; 4: 413.

22. Salech F, Valderrama S, Nervi B, Rodriguez JC, Oksenberg D. Thalidomide for the treatment of metastatic hepatic epithelioid hemangioendothelioma: a case report with a long term follow-up. Ann Hepatol 2011; 10: 99-102.

23. Ye B, Li W, Feng J, Shi JX, Chen Y, Han BH. Treatment of pulmonary epithelioid hemangioendothelioma with combination chemotherapy: Report of three cases and review of the literature. Oncol Lett 2013; 5: 1491-1496.

\section{*Correspondence to}

Wei Liu

Department of Medical Oncology

The Fourth Hospital of Hebei Medical University

PR China 